\title{
Perfil do uso de agentes biológicos no tratamento da artrite reumatoide: experiência do Hospital Universitário Walter Cantídio
}

\section{Usage profile of biological agents on rheumatoid arthritis treatment: experience of the brasilian University Hospital Walter Cantídio}

Raquel Telles Quixadá Lima1. Mailze Campos Bezerra². Ádilla Thaysa Mendes Ribeiro³. Marta Maria das Chagas Medeiros ${ }^{4}$.

1 Reumatologista pela Universidade Federal do Ceará (UFC), Hospital Universitário Walter Cantídio, Fortaleza, Ceará, Brasil. 2 Médica Assistente do Serviço de Reumatologia do Hospital Universitário Walter Cantídio, Fortaleza, Ceará, Brasil. 3 Estudante de Medicina da Universidade Federal do Ceará (UFC), Fortaleza, Ceará, Brasil. 4 Professora titular da Faculdade de Medicina da Universidade Federal do Ceará (UFC), Fortaleza, Ceará, Brasil.

\section{RESUMO}

O tratamento da artrite reumatoide (AR) visa prevenir dano articular irreversível e inclui o uso de drogas sintéticas e biológicas. O objetivo deste estudo foi avaliar o perfil do uso de imunobiológicos na artrite reumatoide, no serviço de reumatologia do HUWC. Este estudo observacional, retrospectivo e transversal, identificou 131 pacientes com AR, em uso atual ou nos últimos 5 anos de agentes biológicos. Foram coletados, em entrevista e revisão de prontuário, dados demográficos, clínicos e terapêuticos, incluindo a presença de eventos adversos e infecções relacionadas às drogas prescritas. Nossa amostra se constituiu de subgrupo de pacientes com doença grave e baixo nível socioeconômico. Os imunobiológicos mais utilizados foram os anti-TNF. Cerca de metade dos pacientes apresentou screening positivo para TB latente. O perfil de uso dos agentes biológicos no tratamento dos pacientes com diagnóstico de AR encontra-se em concordância com as recomendações clínicas vigentes.

Palavras-chave: Artrite reumatoide. Terapêutica. Agentes antirreumáticos. Terapia biológica. Tuberculose latente.

\section{ABSTRACT}

Rheumatoid arthritis (RA) treatment is intended to prevent irreversible joint damage and includes the use of synthetic and biologic drugs. The aim of this study was to evaluate the profile of biologic agents use in rheumatoid arthritis in a single brasilian center of rheumatology. This observational, retrospective and cross-sectional study identified 131 patients with RA, in actual or previous use in the last five years of biologic agents. There were collected in an interview and chart review, demographic, clinical and therapeutic data, including the presence of adverse events and infections related to prescription drugs. Our sample consisted of subgroup of patients with severe disease and low socioeconomic status. The biologic drugs most used were the anti-TNF. About half of the patients had positive screening for latent TB. The usage profile of biological agents in the treatment of RA diagnosed patients is in line with current clinical recommendations.

Keywords: Rheumatoid arthritis. Therapeutics. Antirheumatic agentes. Biological therapy. Latent tuberculosis.

Autor correspondente: Raquel Telles Quixadá Lima. Avenida Abolição, 3049, apto 601, Bairro Meireles , Fortaleza, Ceará. CEP: 60165-081. Telefone: +55 85 31030334/999225626. E-mail: raqueltquixada@gmail.com

Conflito de interesses: Não há qualquer conflito de interesses por parte de qualquer um dos autores.

Recebido em: 17 Jun 2015; Revisado em: 08 Jul 2015; Aceito em: 13 Jul 2015. 


\section{INTRODUÇÃO}

Artrite reumatoide (AR) é uma doença inflamatória crônica autoimune, caracterizada por poliartrite aditiva e simétrica com acometimento articular periférico preferencial de pequenas articulações das mãos, punhos e pés. Estima-se que sua prevalência seja de $0,5-1 \%$ da população, com predomínio no sexo feminino, entre a $4^{\mathrm{a}}$ e $6^{\mathrm{a}}$ décadas. ${ }^{1}$

O manejo da artrite reumatoide baseia-se na instituição precoce de drogas modificadoras do curso da doença (DMCD) com o objetivo de controlar a atividade da doença, melhorar a qualidade de vida e o desfecho funcional do paciente, além de prevenir dano articular irreversível..$^{2-4}$

Dentre as DMCD disponíveis, encontram-se as drogas sintéticas e biológicas. As DMCD sintéticas são as drogas de primeira escolha na artrite reumatoide. ${ }^{1}$ Estas incluem o metotrexate, a leflunomida e a sulfassalazina, além dos antimaláricos para os casos leves.

As DMCD biológicas incluem os inibidores de TNF (adalimumabe, certolizumabe, etanercepte, golimumabe e infliximabe), o inibidor de coestimulação de linfócitos $\mathrm{T}$ (abatacepte), o bloqueador do receptor da IL-6 (tocilizumabe) e o depletor de linfócito B antiCD20 (rituximabe).

Estudos sugerem que o uso de DMCD biológicas na artrite reumatoide esteja associada a aumento no risco relativo de infecções oportunistas. ${ }^{5}$ Neste contexto, para a instituição de terapêutica com agentes biológicos, recomenda-se o rastreio de doenças infecciosas como hepatites B e C, HIV, tuberculose e demais doenças endêmicas a depender do domicílio do paciente. A tuberculose (TB) merece destaque, visto que são descritos casos de reativação da doença na vigência do tratamento imunossupressor. Antes do início do tratamento, deve ser feito screening para TB latente (LTB) com radiografia de tórax, PPD (teste tuberculínico) ou IGRA (teste de liberação de interferon gama) e investigação de contato ou TB prévios. O tratamento para LTB deve ser feito com isoniazida por 6 meses, com pelo menos 30 dias de tratamento precedendo o início do imunobiológico. ${ }^{1}$

Nos últimos anos, observam-se muitos avanços no tratamento da artrite reumatoide. Para os pacientes que não atingiram controle da doença com pelo menos dois esquemas de DMCD sintéticas, sendo um deles a combinação de duas drogas, devese considerar a instituição de DMCD biológicas. ${ }^{1}$ Em casos excepcionais, com doença grave e na presença de fatores de mau prognóstico, pode-se considerar o uso destas drogas como parte da estratégia medicamentosa inicial. A prescrição de imunobiológicos tem se tornado mais acessível com o passar do tempo. Entretanto, estes são medicamentos de alto custo e não isentos de eventos adversos. É importante, portanto, que o médico seja criterioso no momento de sua indicação, para que faça uma escolha individualizada, pesando riscos e benefícios para cada paciente, num dado contexto clínico, para que não atrase nem precipite o momento ideal para sua prescrição.

O objetivo principal deste estudo foi avaliar o perfil do uso de imunobiológicos na artrite reumatoide, em uma coorte de pacientes acompanhados no serviço de reumatologia do Hospital Universitário Walter Cantídio (HUWC), na Universidade Federal do Ceará (UFC).

\section{MATERIAIS E MÉTODOS}

Foram selecionados pacientes com diagnóstico de AR acompanhados no serviço de Reumatologia do HUWC da UFC. Os pacientes receberam o diagnóstico pelos critérios do ACR $1987^{6}$ e do ACR/EULAR 2010, ${ }^{7}$ a depender do ano do diagnóstico. Critérios de inclusão: paciente com diagnóstico de AR, de ambos os sexos, em uso atual ou nos últimos 5 anos de DMCD biológicas. Critérios de exclusão: diagnóstico de outra doença autoimune associada à $\mathrm{AR}$, idade $<18$ anos.

O delineamento do estudo foi retrospectivo e transversal. A coleta de dados realizou-se nos ambulatórios e centro de infusão do HUWC no período de maio/2014 a março/2015. Foram feitas entrevistas para coleta de dados sociodemográficos e revisão de prontuários para coleta de variáveis clínicas, com protocolo padronizado. O estudo foi aprovado pelo Comitê de Ética em Pesquisa do HUWC.

As variáveis demográficas coletadas foram idade, sexo, estado civil, nível de escolaridade, raça e profissão. As variáveis clínicas foram tempo de doença, positividade do fator reumatoide, presença de manifestações extra-articulares, tabagismo, comorbidades associadas à AR, uso de DMCD sintéticas e biológicas.

Com relação às DMCD biológicas foram avaliados: tempo para iniciar a primeira droga biológica após diagnóstico de AR, tempo de uso de cada droga, motivos de suspensão (remissão, evento adverso, não resposta e não aderência), eventos adversos e número de drogas usadas por cada paciente. O Disease Activity Score (DAS28) foi usado para avaliação da atividade da doença na última consulta ambulatorial. Foi considerada doença em remissão se DAS2 $8 \leq 2,6$ e atividade leve se DAS28 $>2,6 \mathrm{e} \leq 3,2 .{ }^{8} \mathrm{O}$ registro da última consulta de valores do hematócrito, hemoglobina, velocidade de hemossedimentação (VHS) e proteína C Reativa (PCR) em $\mathrm{mg} / \mathrm{dL}$ foram retirados dos prontuários. Dados relativos ao screening para LTB e uso de isoniazida foram também retirados dos prontuários.

Análise estatística: A apresentação dos dados demográficos, clínicos e laboratoriais foi por meio de média (desvio padrão), mediana (intervalo interquartil) e percentagem. O banco de dados utilizado e o programa estatístico foi o Stata versão 9.0.

\section{RESULTADOS}

Em fevereiro de 2015, existiam 555 pacientes cadastrados na farmácia do HUWC para recebimento de DMCD com diagnóstico de artrite reumatoide. Destes, 191 para DMCD biológicas, representando $34,4 \%$ da população de pacientes com AR em seguimento no HUWC. 
Dos 191 pacientes em uso de DMCD biológicas, conseguimos estudar 131 pacientes que compareceram no período do estudo ao ambulatório ou ao centro de infusão $(68,8 \%)$. A maioria era do sexo feminino (92,3\%), com idade média de $55.1+12.2$ anos. O tempo médio de doença foi de $11,3+6,5$ anos. $\mathrm{O}$ fator reumatoide foi positivo em $86,6 \%$ dos pacientes e $12,4 \%$ apresentavam manifestações extra-articulares. A amostra se caracterizou, em sua maioria, por baixo nível de escolaridade $(62,7 \%$ dos pacientes tinham até ensino fundamental completo) e cor da pele não branca (82,9\%). Foi verificado tabagismo em $48 \%$ dos pacientes e as comorbidades mais comuns foram: dislipidemia $(42,6 \%)$, hipertensão arterial $(36,4 \%)$, osteoporose $(30,2 \%)$ e diabetes melitus $(17,8 \%)$ (Tabela 1).

Tabela 1. Características sociodemográficas e clínicas de 131 pacientes com diagnóstico de Artrite Reumatoide que fizeram ou fazem uso de agentes biológicos no serviço de Reumatologia do HUWC-UFC.

\begin{tabular}{lc}
\hline Característica & Resultad \\
\hline Idade (média \pm DP) (mínima - máxima) & $55,1 \pm 12$ \\
Sexo feminino (\%) (Sexo feminino : sexo masculino) & $92,3(12$ \\
Estado civil (\%) & \\
Solteiro & 22,4 \\
Casado & 60,0 \\
Separado & 5,6 \\
Viúvo & 12,0
\end{tabular}

Nível escolar (\%)

Analfabeto/alfabetizado

15,1

$1^{\circ}$. Grau

47,6

$2^{\circ}$. Grau

27,0

Superior

Raça (\%)

Branca

17,1

Morena

32,5

Negra

8,1

Parda

Tempo doença em anos (média \pm DP) (mínimo - máximo)

$11,3 \pm 6,5(1,5-36)$

Presença manifestações extra-articulares (\%)

12,4

Fator reumatoide positivo $(0 \%)$

86,6

Tabagismo (\%)

48,0

Uso de corticoide na última avaliação (\%)

72,5

Presença de comorbidades (\%)

Diabetes melitus

Doenças cardíacas

1,5

Hipotireoidismo

7,0

Dislipidemia

42,6

Osteoporose

30,2

Hipertensão arterial 
As drogas sintéticas mais utilizadas foram o metotrexate, seguido pela leflunomida. A associação de drogas sintéticas mais comumente observada foi a de metotrexate e leflunomida em $69,6 \%$ dos pacientes, seguida por metotrexate e antimaláricos em 14,3\%, (Tabela 2).

O tempo médio para iniciar tratamento com primeira DMCD biológica foi de 8,2 + 6,2 anos, sendo as drogas mais frequentemente utilizadas nesta coorte os anti-TNF (infliximabe 56,5\%, etanercepte $45,8 \%$ e adalimumabe $32,1 \%$ ), seguidos por abatacepte $15,3 \%$, tocilizumabe $13,8 \%$, e rituximabe $8,4 \%$. O número médio de biológicos utilizados por paciente foi de 1,72+1,03 (mínimo: 1; máximo: 5). A classe de biológicos escolhida mais comumente como $1^{\text {a }}$ opção foi o anti-TNF sendo infliximabe $(53,4 \%)$, etarnecepte
$(23,7 \%)$ e adalimumabe $(17,5 \%)$; como segunda opção foram abatacepte $(14,3 \%)$ e rituximabe $(5,3 \%)$ e como terceira opção o abatacepte $(26,9 \%)$, tocilizumabe $(19,2 \%)$ e rituximabe $(19,2 \%)$, (Tabelas 3 e 4$)$.

Os principais eventos adversos relacionados às DMCD biológicas foram infecções $24,3 \%$, seguidos de urticária $12,1 \%$ e reações infusionais $8,6 \%$.

A taxa de suspensão de droga em algum momento do tratamento foi de $81,8 \%$ para o rituximabe, seguido de $65 \%$ para adalimumabe, $64,8 \%$ para infliximabe e $46,7 \%$ para etarnecepte. As causas de suspensão de biológicos mais comuns foram não resposta e evento adverso, (Tabela 5).

Tabela 2. Perfil de uso das drogas sintéticas em 131 pacientes com diagnóstico de Artrite Reumatoide que usaram também agentes biológicos acompanhados no HUWC-UFC.

\begin{tabular}{ll} 
Uso de drogas modificadoras da doença sintéticas (\%) & 100 \\
Antimaláricos & 39,2 \\
Metotrexato & 96,9 \\
Sulfassalazina & 14,6 \\
Ciclosporina & 2,3 \\
Leflunomida & 93,7 \\
\hline Combinação de drogas sintéticas (\%) * & 76,6 \\
Metotrexato + leflunomida & 69,6 \\
Antimaláricos + metotrexato & 14,3 \\
Antimaláricos + metotrexato + sulfassalazina & 5,4 \\
\hline Tempo de uso das drogas sintéticas em anos (média \pm DP) (mediana/ IIQ) & \\
Antimaláricos & $3,5 \pm 3,5(2,4 / 1-4)$ \\
Metotrexato & $6,9 \pm 4,6(6 / 4-9,6)$ \\
Sulfassalazina & $2,2 \pm 2,1(1,7 / 0,8-2,3)$ \\
Leflunomida & $3,4 \pm 2,4(3,2 / 1,4-4,9)$ \\
\hline
\end{tabular}

*: total de 112 associações.

Tabela 3. Perfil de uso das drogas biológicas em 131 pacientes com diagnóstico de Artrite Reumatoide acompanhados no HUWC-UFC.

\begin{tabular}{ll}
\hline Tempo (anos) para iniciar o primeiro biológico após diagnóstico & \\
(média \pm DP) & $8,2 \pm 6,2$ \\
(mediana/ IIQ) & $(6,8 / 3,6-11,2)$ \\
\hline Uso de drogas biológicas (\%) & \\
Anti TNF-alfa: & 56,5 \\
$\quad$ Infliximabe & 32,1 \\
$\quad$ Adalimumabe & 45,8 \\
$\quad$ Etanercepte & 1,8 \\
$\quad$ Certolizumabe & 0,8 \\
$\quad$ Golimumabe & \\
Inibidor da coestimulação Linfócito T & 15,3 \\
$\quad$ Abatacepte &
\end{tabular}




\section{Uso de drogas biológicas (\%)}

Anti CD20

Rituximabe

Anti-IL6R

Tocilizumabe

13,8

Tempo de uso das drogas biológicas (meses) (média \pm DP) (mediana/ IIQ)

Infliximabe

$20,0 \pm 17,8(14 / 7-30)$

Adalimumabe

$15,3 \pm 13,7(9,5 / 7-20)$

Etanercepte

$25,0 \pm 19,7(18,5 / 7-42)$

Certolizumabe

$7,5 \pm 2,1(7,5 / 6-9)$

Golimumabe

6

Abatacepte

$11,3 \pm 14,9(4 / 1-14)$

Rituximabe

$11,2 \pm 15,8(6 / 1-15)$

Tocilizumabe

$6,3 \pm 6,6(4 / 1-10)$

Suspensão da droga em algum momento da doença (\%)

Suspensão

Infliximabe

64,8

Adalimumabe

65,0

Etanercepte

46,7

Certolizumabe

0

Golimumabe

0

Abatacepte

30,0

Rituximabe

81,8

Tocilizumabe

11,1

Eventos adversos dos biológicos (\%)

Pacientes que apresentaram evento adverso

$\begin{array}{ll}\text { Principais eventos adversos } & 42,7\end{array}$

$\begin{array}{lr}\text { Reações infusionais } & 8,6\end{array}$

Infecções 24,3

$\begin{array}{ll}\text { Urticária } & 12,1\end{array}$

Número de biológicos usados por paciente

(média \pm DP)(mediana/ IIQ)

$1,72 \pm 1,03(1 / 1-2)$

\begin{tabular}{ll}
\hline Taxa de remissão/ atividade leve (DAS28 $\leq \mathbf{3 . 2})$ na última avaliação (\%)* & $22,5 / 32,0$ \\
\hline Esquemas de tratamento na última avaliação: & 76,0 \\
DMCD sintética + DMCD biológica+ (\%) & 16,8 \\
MTX e/ou Leflunomida + infliximabe & 23,2 \\
MTX e/ou Leflunomida + etanercepte & 13,6 \\
MTX e/ou Leflunomida + adalimumabe & 7,2 \\
MTX e/ou Leflunomida + abatacepte & 12,0 \\
MTX e/ou Leflunomida + tocilizumabe & 0,8 \\
MTX e/ou Leflunomida + golimumabe & 0,8 \\
MTX e/ou Leflunomida + certolizumabe & 20,0 \\
Somente DMCD biológica (\%) & 3,2 \\
Infliximabe & 4,8 \\
Etanercepte & 0,0 \\
Adalimumabe & 6,4 \\
Tocilizumabe & 5,6 \\
Abatacepte & \\
\hline
\end{tabular}

*: Total de 103 pacientes com DAS28 registrado no prontuário; +: com ou sem prednisona; DMCD: droga modificadora doença. 
Tabela 4. Percentual de uso do biológico como $1^{\mathrm{a}}, 2^{\mathrm{a}}$ e $3^{\mathrm{a}}$ opção no tratamento dos pacientes com diagnóstico de Artrite Reumatoide acompanhados no HUWC-UFC.

\begin{tabular}{|c|c|c|c|}
\hline Biológicos & $\begin{array}{l}1^{\mathrm{a}} \text { opção } \\
(\mathrm{n}=131)^{*}\end{array}$ & $\begin{array}{l}2^{\mathrm{a}} \text { opção } \\
(\mathrm{n}=56)^{+}\end{array}$ & $\begin{array}{l}3^{\mathrm{a}} \text { opção } \\
(\mathrm{n}=\mathbf{2 6})^{\S}\end{array}$ \\
\hline Infliximabe & 53,4 & 8,9 & 0,0 \\
\hline Adalimumabe & 17,5 & 26,8 & 11,5 \\
\hline Etarnecepte & 23,7 & 41,1 & 23,1 \\
\hline Abatacepte & 1,5 & 14,3 & 26,9 \\
\hline Rituximabe & 0,0 & 5,3 & 19,2 \\
\hline Tocilizumabe & 2,3 & 1,8 & 19,2 \\
\hline Golimumabe & 0,7 & 0,0 & 0,0 \\
\hline Certolizumabe & 0,7 & 0,0 & 0,0 \\
\hline
\end{tabular}

*: 131 pacientes usaram, no mínimo, 1 agente biológico; +: 56 pacientes usaram, pelo menos, 2 agentes biológicos; $\$$ : 26 pacientes usaram, pelo menos, 3 agentes biológicos.

Tabela 5. Principais motivos para suspensão* das drogas biológicas em 131 pacientes com diagnóstico de Artrite Reumatoide acompanhados no HUWC-UFC.

\begin{tabular}{|c|c|c|c|c|}
\hline Drogas & Remissão (\%) & Evento adverso (\%) & Não resposta (\%) & Não aderência (\%) \\
\hline Infliximabe & 1,9 & 39,6 & 54,7 & 3,8 \\
\hline Adalimumabe & 3,6 & 17,8 & 67,8 & 10,7 \\
\hline Etanercepte & 0,0 & 24,1 & 66,6 & 3,4 \\
\hline Certolizumabe & - & - & - & - \\
\hline Golimumabe & - & - & - & - \\
\hline Abatacepte & 0,0 & 16,6 & 66,7 & 0,0 \\
\hline Rituximabe & 12,5 & 25,0 & 62,5 & 0,0 \\
\hline Tocilizumabe & 0,0 & 50,0 & 50,0 & 0,0 \\
\hline
\end{tabular}

*Alguns pacientes apresentaram mais de 1 motivo.

Na última avaliação, a maioria dos pacientes (76\%) estava usando alguma DMCD biológica associada a uma DMCD sintética, sendo que os esquemas terapêuticos mais utilizados incluíam metotrexate ou leflunomida em associação, respectivamente, a etanercepte $(23,2 \%)$, infliximabe $(16,8 \%)$, adalimumabe $(13,6 \%)$ e tocilizumabe $(12,0 \%)$. A frequência de pacientes utilizando imunobiológicos em monoterapia foi de $20 \%$, sendo o tocilizumabe $(6,4 \%)$ e abatacepte $(5,6 \%)$ os mais usados. A taxa de remissão ou atividade leve (DAS $28<$ 3,2 ) neste momento foi de $32 \%$ (Tabela 3). O DAS 28 médio registrado foi $4,18+1,68$.

Dos 128 pacientes em que encontramos dados no prontuário sobre screening para LTB, 53,1\% utilizaram isoniazida. Destes, $73,4 \%$ tinham PPD > $5 \mathrm{~mm}, 23,3 \%$ alterações radiológicas sugestivas e $53,1 \%$ epidemiologia positiva.

\section{DISCUSSÃO}

Os pacientes que compõem esta coorte são provavelmente um subgrupo de doença articular mais grave, visto que são aqueles não obtiveram controle de atividade de doença com as DMCD sintéticas e, portanto, tiveram indicação de terapia com imunobiológicos. Talvez por este motivo, observamos frequência maior de fator reumatoide positivo $86,6 \%$ do que o encontrado na artrite reumatoide estabelecida, que varia de 60 a $80 \% .^{9}$

Constatamos exposição ao tabagismo em quase metade de nossa amostra. Estudos sugerem que o tabagismo associa-se tanto ao aumento da susceptibilidade ao desenvolvimento de AR em indivíduos geneticamente predispostos, quanto a pior resposta clínica à terapêutica, em especial aos agentes antiTNF. ${ }^{10} \mathrm{O}$ fumo promove inflamação persistente devido ao stress oxidativo, estado pro-inflamatório, produção de autoanticorpos e efeitos epigenéticos implicados na autoimunidade da artrite reumatoide, devendo ser desencorajado em pacientes portadores de AR.

Observamos um longo tempo médio para início de terapia imunobiológica. Talvez isto se justifique pelo longo tempo de doença dos pacientes de nossa amostra. Desta forma, no início de seu seguimento, as drogas imunobiológicas talvez não estivessem disponíveis para prescrição médica. No HUWC, inicialmente, a partir de julho de 2004, estavam liberados para uso apenas infliximabe, adalimumabe e etanercepte. A partir de setembro de 2013, foi liberado pelo ministério da saúde mais dois anti-TNF (certolizumabe e golimumabe), além do tocilizumabe, abatacepte e rituximabe. Este fato pode ter contribuído significativamente para a casuística desta coorte, que tem maior número de pacientes em uso de agentes anti-TNF tradicionais. As demais drogas foram empregadas predominantemente em casos de diagnóstico mais recente ou de falha a mais de um anti-TNF. Outros motivos que podem ter contribuído para este longo tempo são o predomínio de baixo nível socioeconômico em nossa amostra e as dificuldades estruturais de nosso sistema de saúde, fatores citados em estudos prévios ${ }^{11}$ como associados à menor probabilidade de início de tratamento com DMCD biológica. 
Apesar de o consenso brasileiro de 2012 ainda recomendar os agentes anti-TNF como biológicos de primeira escolha por conta da maior experiência cronológica dos prescritores com seu uso, o consenso europeu de 2014 considera que tanto os anti-TNF quanto o tocilizumabe e o abatacepte sejam adequados como primeira droga biológica a ser prescrita, a critério clínico. O rituximabe, entretanto, deve ser reservado para pacientes não respondedores à terapia com anti-TNF ou para aqueles virgens de tratamento com DMCD biológicas em situações especiais como: diagnóstico de doença linfoproliferativa ou neoplasia sólida nos últimos 5 anos, história prévia de doença desmielinizante ou TB latente com contraindicação à quimioprofilaxia. ${ }^{2,12}$

A prescrição de imunobiológicos deve ser feita em combinação com droga sintética, de preferência o metotrexate, ${ }^{13}$ exceto se for contraindicado. Evidências mostram que a associação de metotrexate com DMCD biológica é a que está menos associada à suspensão da medicação por evento adverso, ${ }^{14}$ além de seus efeitos sinérgicos antinflamatórios e de aumento da tolerância imunológica aos anticorpos monoclonais. É relevante ressaltar que o tocilizumabe pode ser utilizado em monoterapia quando necessário, porém mesmo esta droga tem uso combinado preferencial. ${ }^{2,13}$ Informações de registros do uso de imunobiológicos indicam que até 30\% dos pacientes fazem uso de DMCD biológicas em monoterapia. ${ }^{15}$ Em nossa coorte, encontramos taxa de monoterapia de $20 \%$ na última consulta, sendo o tocilizumabe o mais usado isoladamente sem droga sintética.

As causas mais comuns de suspensão dos imunobiológicos foram a ocorrência de não resposta, seguida de eventos adversos, conforme o descrito no registro brasileiro do uso de imunobiológicos. ${ }^{16}$ Os eventos adversos mais descritos para a DMCD biológicas são as infecções e as reações infusionais, o que está de acordo com nossos resultados.

Mais da metade dos pacientes de nossa amostra utilizaram a isoniazida para tratamento de LTB. Notamos uma alta porcentagem de pacientes com epidemiologia de contato com TB $(50 \%)$ e PPD $>5 \mathrm{~mm}(75 \%)$. Foi registrado um caso de reativação de TB extrapulmonar (peritoneal) nesta coorte. Estudos mostram o aumento no risco relativo de infecção por TB em pacientes tratados com imunobiológicos comparado à população geral (OR 3,73; IC 95\%: 1,72-8,13) $)^{5}$ e àqueles em tratamento com drogas sintéticas (OR 2,67; IC 95\%: 2,12$3,34) .{ }^{17}$ Risco maior se associa aos anti-TNF, notadamente tipo anticorpo monoclonal no primeiro ano de tratamento. Sabe-se que o tratamento de LTB com isoniazida diminui o risco de TB em apenas $60 \% .{ }^{18}$ Estes dados enfatizam a importância

\section{REFERÊNCIAS}

1. Mota LM, Cruz BA, Brenol CV, Pereira IA, Rezende-Fronza LS, Bertolo MB, et al. Brazilian Society of Rheumatology Consensus for the treatment of rheumatoid arthritis. Rev Bras Reumatol. 2012;52(2):152-74.

2. Smolen JS, Landewé R, Breedveld FC, Buch M, Burmester G, DLougados M, et al. EULAR recommendations for the management de investigar todos os pacientes para LTB antes do início da terapia com imunobiológicos, além de vigiar surgimento de infecção ativa na vigência do tratamento.

Em nosso estudo, observamos um DAS28 médio na última consulta de 4,18 , que traduz atividade de doença moderada. A taxa de remissão foi de $22,5 \%$ e a de atividade leve, $32 \%$. Em estudos de coorte no Canadá e na Holanda são descritas taxas de remissão de $35 \%$ e $27 \%$, respectivamente. ${ }^{19}$

Deste fato surgem alguns questionamentos: nossos pacientes são não respondedores por terem doença articular mais grave? Existe adesão à medicação prescrita? Há compreensão pelos pacientes quanto à autoavaliação na escala visual analógica de dor (EVA) utilizada no DAS 28? Segundo Chu LH et al, alguns fatores estão associados à pior adesão ao tratamento. Entre eles estão raça afroamericana (OR 1,49; IC 95\%: 1,032,71 ) e a presença de comorbidades (OR 1,24; IC 95\%: 1,011,57). Por outro lado, uso de corticoides (OR 0,80; IC 95\%: 0,63-0,98) e prática de terapia ocupacional ou fisioterapia concomitantes (OR 0,66; IC 95\%: 0,46-0,93) foram fatores contribuidores para adesão. ${ }^{20} \mathrm{Em}$ nossa amostra, embora a maioria dos pacientes fizesse uso de corticoide, percebemos predominância de baixo nível socioeconômico, raça não branca e alta percentagem de comorbidades associadas. Talvez, estes fatores tenham contribuído para a manutenção de atividade da doença quando medida por métrica internacional padronizada.

\section{CONCLUSÕES}

O uso de imunobiológicos no tratamento da artrite reumatoide tem ficado cada vez mais acessível, para o subgrupo de pacientes não respondedores a DMCD sintéticas. Avaliamos um grupo de pacientes com doença grave e de longo tempo de evolução, com baixo nível socioeconômico em sua maioria. Os biológicos mais utilizados foram os antiTNF. As causas mais comuns para suspensão de imunobiológicos foram reação adversa e não resposta. As principais reações adversas foram infecções e reações infusionais. Na última consulta, a maioria dos pacientes estava em uso de terapia combinada de DMCD sintética e biológica, mas apesar disto, apenas um terço deles atingiu alvo terapêutico. Cerca de metade dos pacientes apresentaram screening positivo para TB latente e fizeram tratamento com isoniazida.

O perfil de uso dos agentes biológicos no tratamento dos pacientes com artrite reumatoide seguidos no serviço de Reumatologia do HUWC-UFC encontra-se em concordância com as recomendações clínicas nacionais e internacionais.

of rheumatoid arthritis with synthetic and biological diseasemodifying antirheumatic drugs: 2013 update. Ann Rheum Dis. 2014;73: 492-509.

3. Mota LM, Cruz BA, Brenol CV, Pereira IA, Fronza LS, Bertolo MB, et al. 2011 Consensus of the Brazilian Society of Rheumatology for diagnosis and early assessment of rheumatoid arthritis. Rev Bras 
Reumatol. 2011;51(3):199-219.

4. Smolen JS, Aletaha D, Bijlsma JW, Breedeveld FC, Boumpas D, Burmester G, et al. Treating rheumatoid arthritis to target: recommendations of an international task force. Ann Rheum Dis. 2010;69(4):631-7.

5. Kourbeti IS, Ziakas PD, Mylonakis E. Biologic therapies in rheumatoid arthritis and the risk of opportunistic infections: a metaanalysis. Clin Infect Dis. 2014;58(12):1649-57.

6. Arnett FC, Edworthy SM, Bloch DA, McShane DJ, Fries JF, Cooper NS, et al. The American Rheumatism Association 1987 revised criteria for the classification of rheumatoid arthritis. Arthritis Rheum. 1988;31(3):315-24.

7. Aletaha D, Neogi T, Silman AJ, Funovits J, Felson DT, Bingham CO 3rd, et al. 2010 Rheumathoid Arthritis classification criteria: an American College of Rheumatology / European League Against Rheumatism collaborative initiative. Ann Rheum Dis. 2010;69(9): 1580-8.

8. Dougados M, Aletaha D, van Riel P. Disease activity measures for rheumatoid arthritis. Clin Exp Rheumatol. 2007;25 Suppl 46:S22-9.

9. Hochberg MC, Silman AJ, Smolen JS, Weinblatt ME, Weisman MH. Rheumatology. 5th ed. Philadelphia: Mosby; 2011. 2228 p.

10. Chang K, Yang SM, Kim SH, Han KH, Park SJ, Shin JI. Smoking and rheumatoid arthritis. Int J Mol Sci. 2014;15(12):22279-95.

11. Yelin E, Tonner C, Kim SC, Katz JN, Ayanian JZ, Brookhart MA, et al. Sociodemographic, disease, health system, and contextual factors affecting the initiation of biologic agents in rheumatoid arthritis: a longitudinal study. Arthritis Care Res (Hoboken). 2014;66(7):980-9.

12. Singh JA, Furst DE, Bharat A, Curtis JR, Kavanaugh AF, Kremer JM, et al. 2012 update of the 2008 American College of Rheumatology recommendations for the use of disease-modifying antirheumatic drugs and biologic agents in the treatment of rheumatoid arthritis. Arthritis Care Res (Hoboken). 2012;64(5):625-39.

13. Ruderman EM. The role of concomitant methotrexate in biologic therapy for rheumatoid arthritis. Bull Hosp Jt Dis. 2013;71 Suppl 1:S29-32.

14. Abasolo L, Leon L, Rodriguez-Rodriguez L, Tobias A, Rosales $Z$, Maria Leal J, et al. Safety of disease-modifying antirheumatic drugs and biologic agents for rheumatoid arthritis patients in real-life conditions. Semin Arthritis Rheum. 2015;44(5):506-13.

15. Emery P, Sebba A, Huizinga TW. Biologic and oral diseasemodifying antirheumatic drug monotherapy in rheumatoid arthritis. Ann Rheum Dis. 2013;72(12):1897-904.

16. Titton DC, Silveira IG, Louzada-Junior P, Hayata AL, Carvalho HM, Ranza R, et al. Brazilian biologic registry: BiobadaBrasil implementation process and preliminary results. Rev Bras Reumatol. 2011;51(2):152-60.

\section{Chiu YM, Lang HC, Lin HY, Yang MT, Fang CH, Yang YW,}

et al. Risk of tuberculosis, serious infection and lymphoma with disease-modifying biologic drugs in rheumatoid arthritis patients in Taiwan. Int J Rheum Dis. 2014;17 Suppl 3:9-19.

18. Tikly M, Hodkinson B, Dheda K. Biologic therapy for Rheumatoid Arthritis in developing countries - a place for non-TNF inhibitors as first-line treatment? Rheumatology (Oxford). 2015;54(2):208-9.

19. Russel AS, Mallon C, Conner-Spady B, MaksymowychW. A biologic pharmacosurveillance program for rheumatoid arthritis: a single-center experience. Clin Rheumatol. 2013;32(6):875-7.

20. Chu LH, Kawatkar AA, Gabriel SE. Medication Adherence and attrition to biologic treatment in rheumatoid arthritis patients. Clin Ther. 2015;37(3):660-6.

\section{Como citar:}

Lima RT, Bezerra MC, Ribeiro AT, Medeiros MM. Perfil do uso de agentes biológicos no tratamento da artrite reumatoide: experiência do Hospital Universitário Walter Cantídio. Rev Med UFC. 2015 jul-dez;55(2):15-22. 